\title{
A D-region conductivity model from EISCAT VHF measurements
}

\author{
K. Schlegel ${ }^{1}$ and M. Füllekrug ${ }^{2}$ \\ ${ }^{1}$ Max-Planck-Institut für Aeronomie, Max-Planck-Str. 2, D-37191 Katlenburg-Lindau, Germany \\ ${ }^{2}$ Institut für Meteorologie und Geophysik, Universität Frankfurt, Feldbergstr. 47, D-60323 Frankfurt/Main, Germany
}

Received: 17 October 2001 - Revised: 18 February 2002 - Accepted: 28 February 2002

\begin{abstract}
An easy-to-use model to evaluate conductivities at high and middle latitudes in the height range $70-100 \mathrm{~km}$ is presented. It is based on electron density profiles obtained with the EISCAT VHF radar during 11 years and on the neutral atmospheric model MSIS95. The model uses solar zenith angle, geomagnetic activity and season as input parameters. It was mainly constructed to study the properties of Schumann resonances that depend on such conductivity profiles.
\end{abstract}

Key words. Meteorology and atmospheric dynamics (middle atmospheric dynamics) - Ionosphere (modeling and forecasting; ionosphere-atmosphere interaction)

\section{Introduction}

The motivation for this work was the need for easy-touse, but reliable conductivity profiles in the D-region for Schumann-resonance (SR) studies. Schumann resonances are resonant electromagnetic waves in the Earth-ionosphere cavity with a fundamental frequency of about $8 \mathrm{~Hz}$ and higher order modes. They have been predicted and theoretically discussed by Schumann (1952). In the following decades they were extensively studied (e.g. Sentman, 1995, for a recent review). It is commonly assumed that lightning discharges from global thunderstorm activity are the main excitation sources. SR can be fully characterized by three parameters: amplitude, center frequency and spectral width (Füllekrug, 1995), the latter is a measure of the damping of the waves. Current theories (e.g. Sentman, 1995 and references therein) show that at least two of these parameters, the center frequency and the spectral width strongly depend on the conductivity of the lower D-region, i.e. the upper boundary of the Earth-ionosphere wave guide. Reliable conductivity profiles are, therefore, important to estimate these parameters and in turn to describe the behavior of SR.

During the last 20 years only constant profiles have been used which were derived from rocket measurements obtained in the seventies (compiled by Tran and Polk, 1979), with no allowance for seasonal or geomagnetic variations. Apart

Correspondence to: K. Schlegel (schlegel@linmpi.mpg.de) from a recent WWW-based conductivity model at the WDC for Geomagnetism in Kyoto (see Sect. 5), which so far did not come to the attention of the SR community, our model is the first attempt to improve the situation. It was required that the model should depend on only a few key quantities which determine the temporal variation and only on a minimal number of parameters to characterize the profiles. In the first place, the purpose of the model was to show the variations of the conductivity profiles during changes in geophysical conditions, rather than to obtain a most realistic profile for a particular day and time. Although SR is a global phenomenon, the "local" conductivities at high latitudes (of both hemispheres) and their seasonal and geomagnetic variations are important. These boundary conditions led to the construction of the model from EISCAT data described in the following. The application of the model to SR physics is beyond the scope of this paper; a separate contribution is in preparation.

The following section describes the processing of the electron density profiles, Sect. 3 describes the conductivity calculation, Sect. 4 describes the construction of the model, and Sect. 5 contains examples of typical results and a discussion.

\section{EISCAT CP-6 electron densities}

EISCAT is a state-of-the-art incoherent scatter system, comprising several different radars (Rishbeth and van Eyken, 1993). For our purpose data from the VHF (224 MHz) radar are convenient, since this radar yields height profiles of the electron density in the D- and lower E-regions with a spatial resolution of about one kilometer and a temporal resolution of a few minutes. A pulse-to-pulse correlation is used to resolve the narrow spectra recorded in this altitude range (Turunen, 1986). From the measured electron density profiles, the conductivities are calculated using the MSIS95 model for neutral densities.

We use for our model electron densities obtained with the EISCAT Common Programme 6. It provides the electron density between 70 and more than $100 \mathrm{~km}$ altitude with a height resolution of $1.05 \mathrm{~km}$ measured in the vertical direction. Since densities above $100 \mathrm{~km}$ are not important for our purpose, we select only the first 30 height gates, ranging from 

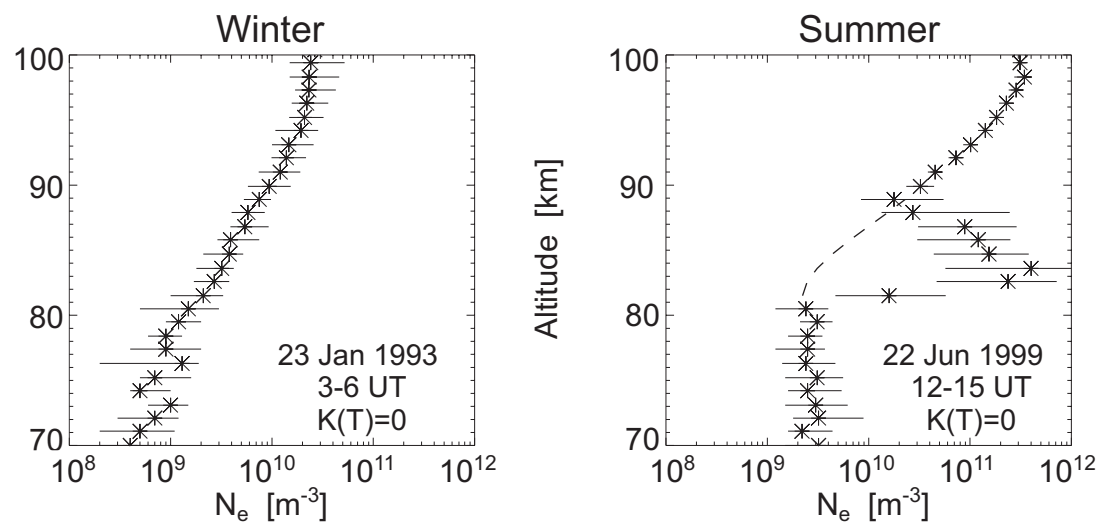

Fig. 1. Sample 3-h median profiles from EISCAT CP-6 measurements for typical winter (night) and summer (day) conditions. In the summer profile the interpolation of the electron density during PMSE events is demonstrated.

70.0 to $100.4 \mathrm{~km}$. The post integration of the measured profiles is usually 5 minutes. Between 1990 and 2000, CP-6 was operating on 102 days.

As a first step we calculated median profiles for a time interval of 3 hours in order to obtain temporal coincidence with geomagnetic indices. In total, we obtained reliable data for 572 such median profiles for the above mentioned 102 days, omitting intervals with corrupted data due to low signal-tonoise ratio or system malfunctions. Figure 1 shows two extreme cases of such median profiles, one for a winter night and one for a summer day. The summer data often contained so-called polar mesospheric summer echoes (PMSE). These are coherent echoes and do not indicate enhanced electron densities. Therefore, we logarithmically (spline) interpolated these echoes which is a reliable method, since the PMSE are confined to a relatively narrow height range and enough "real" electron density data points are available above and below the PMSE. The method is illustrated at the summer profile in Fig. 1.

As already mentioned, the 3-hour intervals were chosen to coincide with geomagnetic indices, since the electron density profiles depend heavily on geomagnetic activity. We did not use $\mathrm{Kp}$ but rather the local $\mathrm{K}$ derived from the Troms $\varnothing$ magnetometer (http://geo.phys.uit.no/geomag.html), which is very closely related with the EISCAT measurements. The other parameter strongly affecting the electron density is the solar zenith angle, which was consequently also used to characterize the density profiles.

Figure 2 shows histograms of the distributions of the quantities characterizing the density profiles. In the upper panel the 572 median profiles are separated with respect to the local $K$, thus representing the average geomagnetic conditions in northern Scandinavia. Most of the profiles were recorded during moderately disturbed conditions ( $K=3$ ), less at very quiet times and only very few during strongly disturbed conditions. The middle panel shows the seasonal distribution of the available median profiles which is rather uniform, apart from somewhat less data during spring. The lowest panel characterizes the solar zenith angle $(\chi)$ distribution of the 3$\mathrm{h}$ median profiles. They are binned according to $\cos \chi$ with a bin width of 0.2. The lowest solar zenith angle occurring at
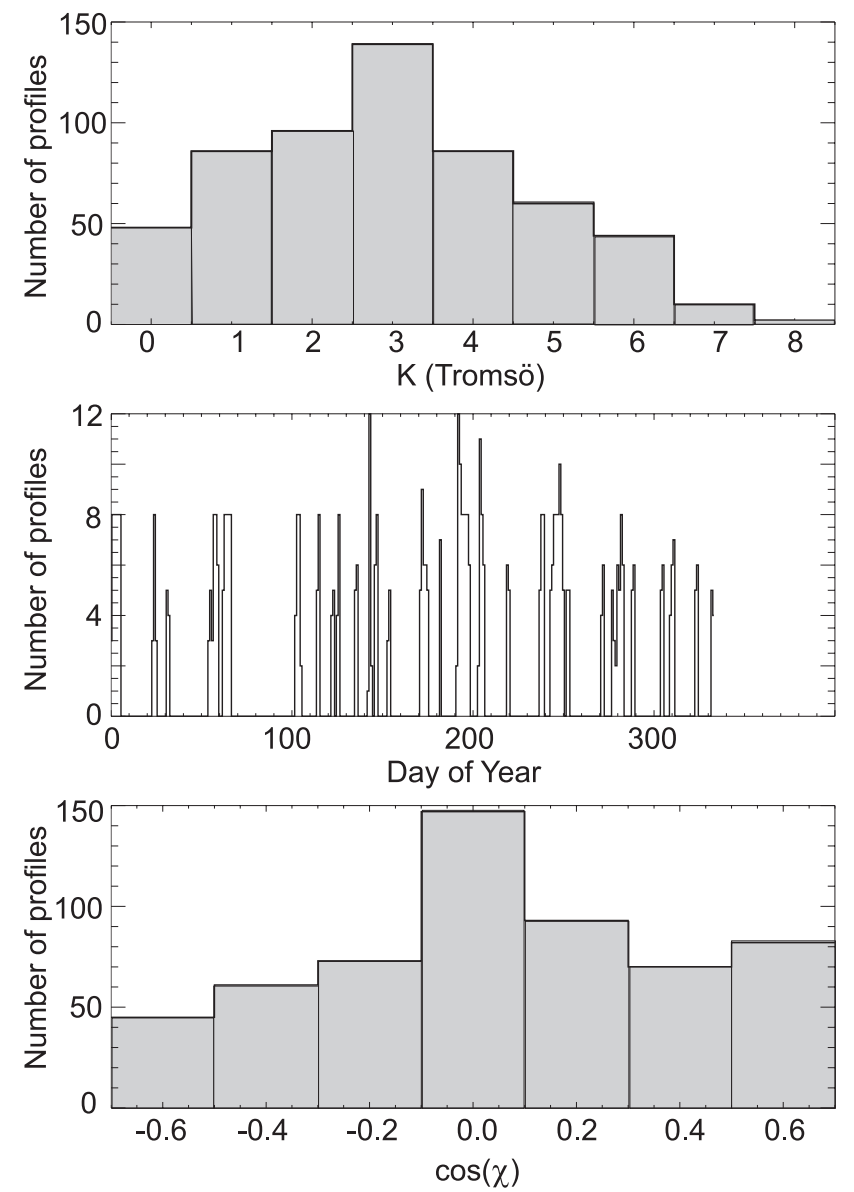

Fig. 2. Distribution of the conditions of the 572 median profile prepared for the model. Upper panel: local geomagnetic index $K$; middle panel: seasonal distribution; lower panel: solar zenith angle distribution (bin size 0.2).

the EISCAT location is $\chi \approx 46^{\circ}$.

We did not separate our set of profiles according to solar cycle variations. The change in particle precipitation during the cycle is sufficiently characterized by $K$; the variability due to solar EUV flux changes is completely negligible at $70 \mathrm{~km}$, and only weak at altitudes between 90 and $100 \mathrm{~km}$. 
It should be mentioned, however, that the D-region conductivity at high latitudes is heavily enhanced during the precipitation of high energy solar protons and electrons occurring, for instance, after solar flares. During such conditions, the behavior of SR is substantially changed (Schlegel and Füllekrug, 1999). These events are quite rare and there are only $2-3$ cases coinciding with EISCAT measurements, which is not enough to be representative for our model.

\section{Conductivities}

Ionospheric conductivities depend strongly on, in addition to electron density $\left(n_{e}\right)$, collision frequencies between neutrals and electrons $\left(v_{e n}\right)$ and ions $\left(v_{i n}\right)$. Since the conductivity is, in principle, a tensor quantity due to the influence of the geomagnetic field, we have the three components:

Pedersen conductivity $\sigma_{p}=\frac{e n_{e}}{B}\left(\frac{\omega_{e} v_{e}}{\omega_{e}^{2}+v_{e n}^{2}}+\frac{\omega_{i} v_{i n}}{\omega_{i}^{2}+v_{i n}^{2}}\right)$,

Hall conductivity $\quad \sigma_{H}=\frac{e n_{e}}{B}\left(\frac{\omega_{e}^{2}}{\omega_{e}^{2}+v_{e n}^{2}}-\frac{\omega_{i}^{2}}{\omega_{i}^{2}+v_{i n}^{2}}\right)$,

parallel conductivity $\quad \sigma_{\|}=e^{2} n_{e}\left(\frac{1}{m_{e} v_{e n}}+\frac{1}{m_{i} v_{i n}}\right)$.

Since $\sigma_{p}<\sigma_{H}<\sigma_{\|}$holds at D-region heights, only the parallel conductivity is important for SR studies (Tran and Polk, 1976) and will be regarded in the following. The collision frequencies depend mainly on neutral densities. We used the following numerical relations (Schunk and Nagy, 1978; Schunk and Walker, 1973):

$$
\begin{aligned}
v_{i n} & =4.34 \cdot 10^{-10} n\left(N_{2}\right)+4.2 \cdot 10^{-10} n\left(O_{2}\right) \\
& +2.44 \cdot 10^{-10} n(O), \\
v_{e n} & =2.33 \cdot 10^{-11} n\left(N_{2}\right) \cdot\left(1-1.21 \cdot 10^{-4} T_{e}\right) \cdot T_{e} \\
& +1.82 \cdot 10^{-10} n\left(O_{2}\right) \cdot\left(1+3.6 \cdot 10^{-2} \sqrt{T_{e}}\right) \cdot \sqrt{T_{e}} \\
& +8.9 \cdot 10^{-11} n(O) \cdot\left(1+5.7 \cdot 10^{-4} T_{e}\right) \cdot \sqrt{T_{e}},
\end{aligned}
$$

where the densities of the major neutral species, $n\left(N_{2}\right)$, $n\left(O_{2}\right)$ and $n(O)$ are in $\mathrm{m}^{-3}$, and $T_{e}=T_{i}=T_{n}$ in $K$. It should be noted that atomic oxygen makes only a very small contribution in this height range.

The neutral densities and the neutral temperature have been taken from the MSIS95 model. Since geophysical parameters such as $A_{p}, \mathrm{~F} 10.7, \chi$, and day of year enter this model as parameters, we have calculated the densities and temperature exactly for the conditions of each 3-hour median profile. Subsequently, the conductivity profiles were calculated using the above relations.

We are aware that the MSIS95 model is not perfect at high latitudes and that more reliable local mesospheric models have been constructed from falling-sphere data (e.g. Lübken, 1999). We compared these season-dependent mass densities with those from MSIS95 and found generally good

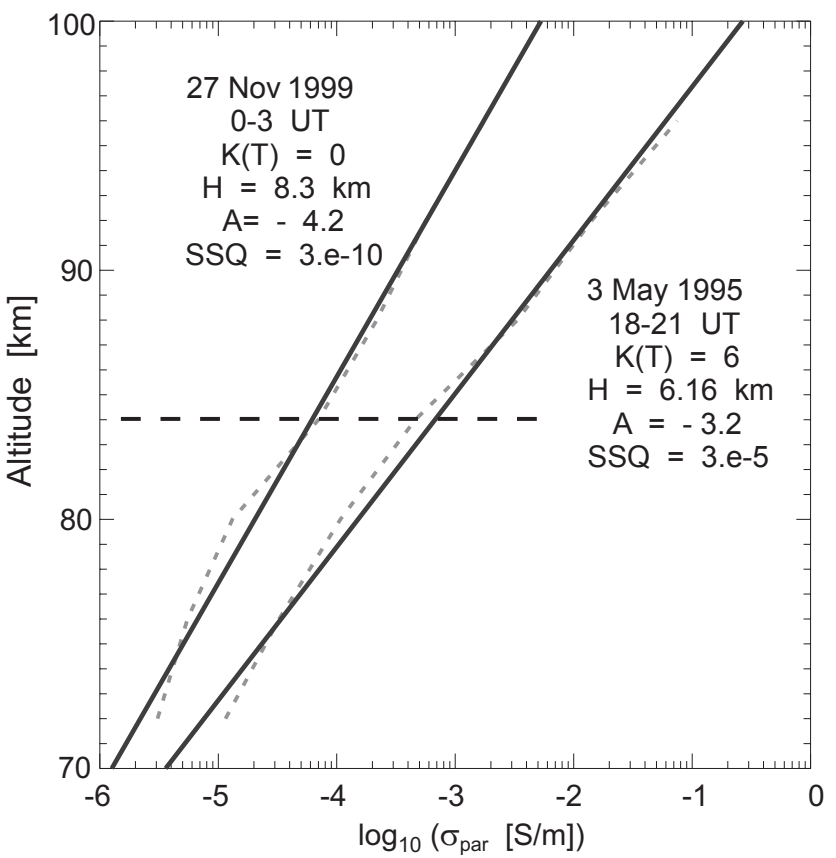

Fig. 3. Conductivity profiles calculated from the CP-6 median electron density profiles (dashed lines) and the log-linear approximation of these profiles by Eq. (4) for two different conditions. The respective model parameters $H$ and $A$ are indicated, together with the geomagnetic conditions and the goodness of the fit (SSQ).

agreement (difference less than 10\%) in the 70-85 km height range. Only above $90 \mathrm{~km}$ did the differences sometimes reach $20 \%$, particularly in spring and fall. For these altitudes, the dependence on $A_{p}$ and $\chi$ becomes increasingly important; this is not accounted for in the Lübken (1999) model, but in MSIS95. Therefore, we retained the MSIS95 model, since it has the additional advantage of being available on-line via the internet and it is widely used. Thus, our results can easily be verified. For the ions, we assumed $m_{i}=31 \mathrm{AMU}\left(50 \% \mathrm{NO}^{+}, 50 \% \mathrm{O}_{2}^{+}\right)$in Eq. (1).

The dashed lines in Fig. 3 represent two examples of such conductivity profiles. The conductivities are given in $\mathrm{S} / \mathrm{m}$ throughout the paper. A careful inspection of all the CP-6 electron density profiles reveals that the measured densities are rarely lower than about $0.6 \times 10^{8} \mathrm{~m}^{-3}$. This value, therefore, probably defines the threshold of the VHF system. At the very low end of our measuring range, the densities may thus be biased towards values above this threshold. In order to mitigate this effect, we omitted the first two height gates of the profiles; the conductivity profiles used in the model thus start at $72 \mathrm{~km}$.

\section{The conductivity model}

In the Introduction we stated that the conductivity model has to be simple and only a minimal number of parameters should be used to characterize the profiles. Therefore, we approximated the calculated conductivity profiles by a linear 

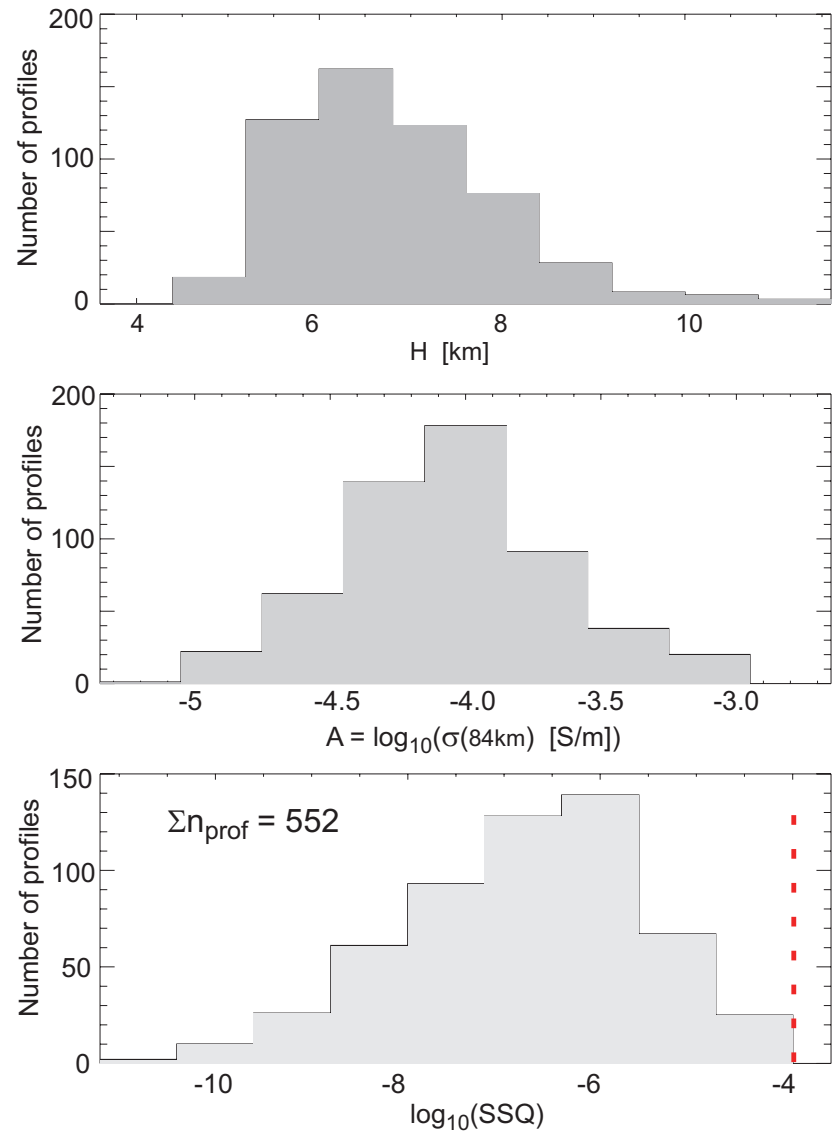

Fig. 4. Histograms of the two input parameters of our model, $H$ and $A$, together with the goodness of the fit (Eq. 4). SSQ has been limited to values $<10^{-4}$, that means that finally 552 profiles have been used for the model.

function in $\log (\sigma)$. Such a function depends on two parameters for which we chose $H$, the scale height of the conductivity profile at $84 \mathrm{~km}$, and $10^{A}$, the conductivity value at $84 \mathrm{~km}$,

$\sigma_{\|}(h)=10^{(h-84 \mathrm{~km}) / H+A}$.

This representation is convenient for SR studies, since the conductivity scale height enters the relevant equations (Füllekrug, 2000).

In Fig. 3, we have plotted the approximated exponential profile as a solid line, together with the values of $H$ and $A$. The approximation by Eq. (4) is certainly not perfect, but it represents a good compromise between accuracy and the required simplicity of the model. Larger deviations usually occur below $75 \mathrm{~km}$ where the radar data may be biased, as mentioned before. In the fit process, therefore, a higher weight was given to the points in the upper part of the profile. The S-shape of many of the electron density profiles (e.g. Fig. 1) is straightened by the strong height dependence of the collision frequency and, therefore, not so pronounced in the conductivity profiles. SSQ gives the reduced sum of squares, generally used as a relative measure of the goodness of the fit.
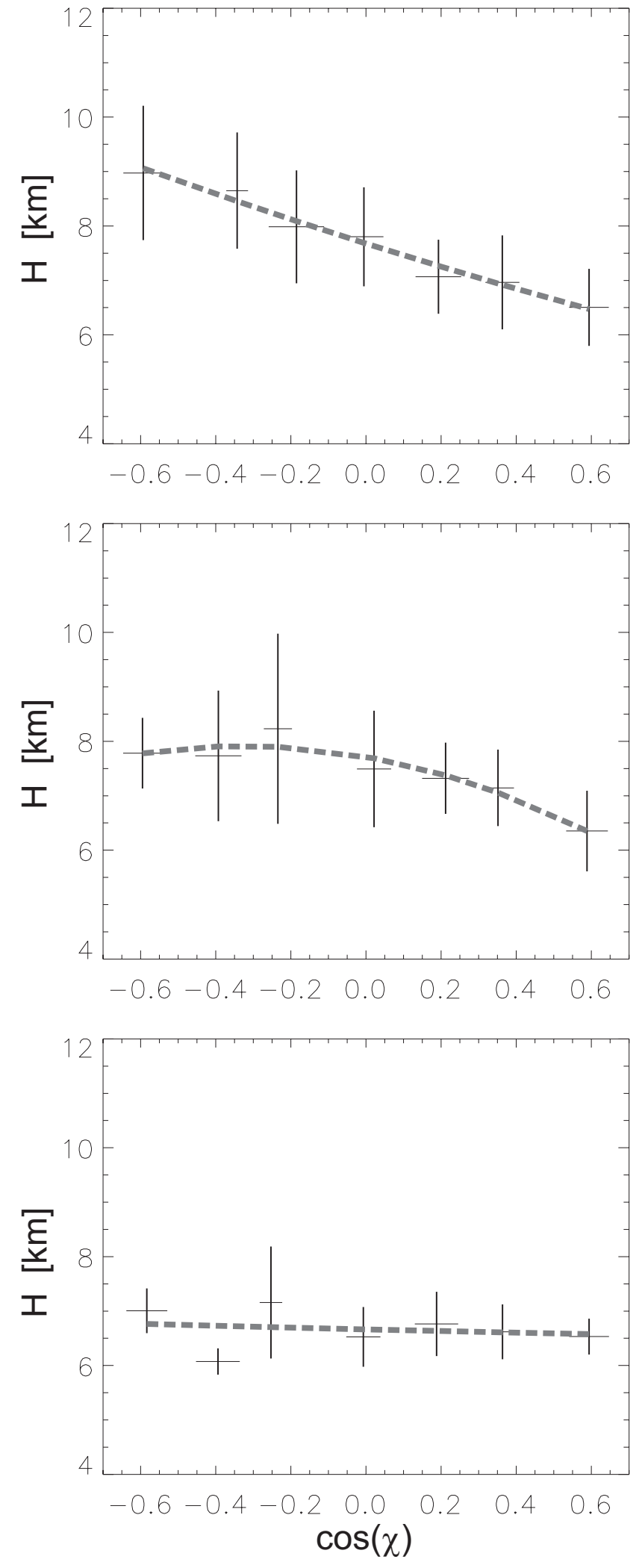

Fig. 5. Dependence of the scale height $H$ on solar zenith angle $(\cos \chi)$ and geomagnetic condition. Upper panel: $K=0,1$, middle panel: $K=3$, lower panel: $K=6,7,8$. 

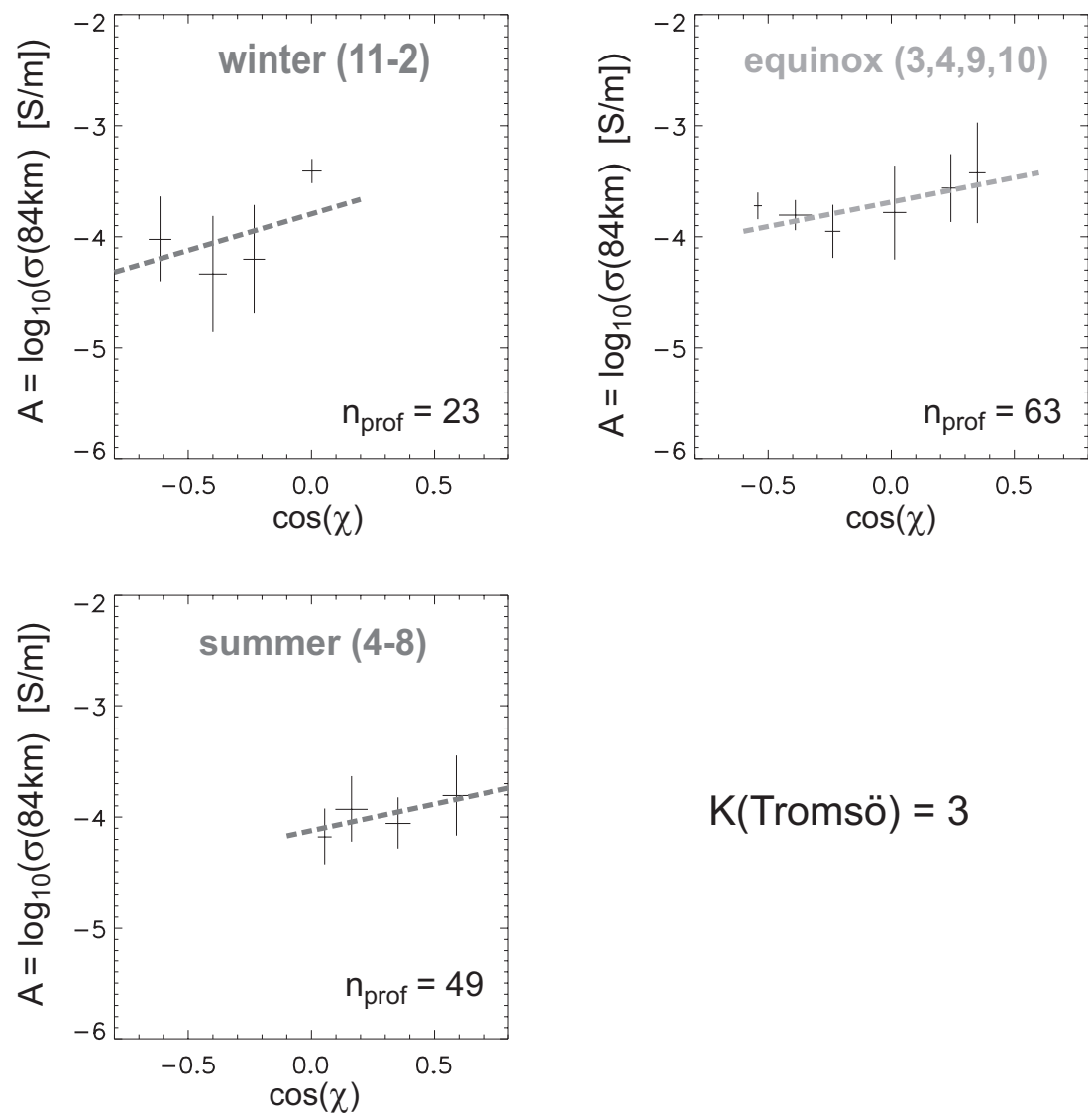

Fig. 6. Dependence of the constant $A$ on solar zenith angle and season for geomagnetic conditions $K=3$.
Figure 4 shows histograms of these three model parameters. From the upper panel it can be seen that the scale height $H$ is mostly in the range of $6-8 \mathrm{~km}$, and the middle panel indicates that the conductivity at $84 \mathrm{~km}$ altitude is mainly between $3 \cdot 10^{-5}$ and $3 \cdot 10^{-4} \mathrm{~S} / \mathrm{m}$. The lowest panel shows the goodness of the fit (4) to the experimental profiles. We set the limit for SSQ to $10^{-4}$, thereby omitting some profiles for which this fit was unacceptable. Thus, 552 conductivity profiles were finally included into our model.

The next step in building our conductivity model was to find reasonable approximations for the dependence of $H$ and $A$ on the main characterizing quantities of the input profiles, namely $K$ and $\cos \chi$.

Figure 5 demonstrates the dependence of the scale height $H$ on the magnetic index $K$ and the solar zenith angle. The crosses indicate the average values of the model input $(H$ from the 552 profiles described above) and their standard deviation in the respective bin, the dashed line represents a quadratic fit, as used in our model. For quiet magnetic conditions (upper panel), $H$ depends strongly on $\cos \chi$; for slightly disturbed conditions, this dependency becomes weaker (middle panel) and for strong magnetic disturbances, $H$ is practically independent of $\cos \chi$ (lower panel). The physics behind this behavior is the following: during small $K$, particle precipitation is absent or low and the corresponding electron density/conductivity profiles are mainly determined by the solar EUV radiation and little affected by enhanced ion- ization. With increasing $\cos \chi$, the profiles become flatter (decreasing $H$ ) in this case, since the solar radiation ionizes stronger above $84 \mathrm{~km}$ than below. With increasing $K$, the additional ionization through particle precipitation becomes more and more important, and finally dominates over the solar radiation. Simultaneously, $H$ becomes smaller (the profiles get flatter, since particle precipitation acts to increase the electron densities/conductivities mainly above $84 \mathrm{~km}$.

For the constant $A$, it turns out that it also depends significantly on the season. This is most probably due to the fact that the MSIS95 neutral densities vary with the day of the year. In order to keep the values in our bins at a reasonable number (statistical significance), we separated the input values of the constant $A$ only for three different seasons, winter (November-February), summer (May-August) and equinox (March, April, September, October). Figure 6 shows as an example the constant $A$ as a function of $\cos \chi$, and these three seasons for a magnetic index $K=3$. Similar linear approximations have been established for other values of $K$.

\section{Examples of model results and discussion}

It was stated in the Introduction that our model should primarily be used to show the variation of the conductivity during changes in the geophysical conditions. 

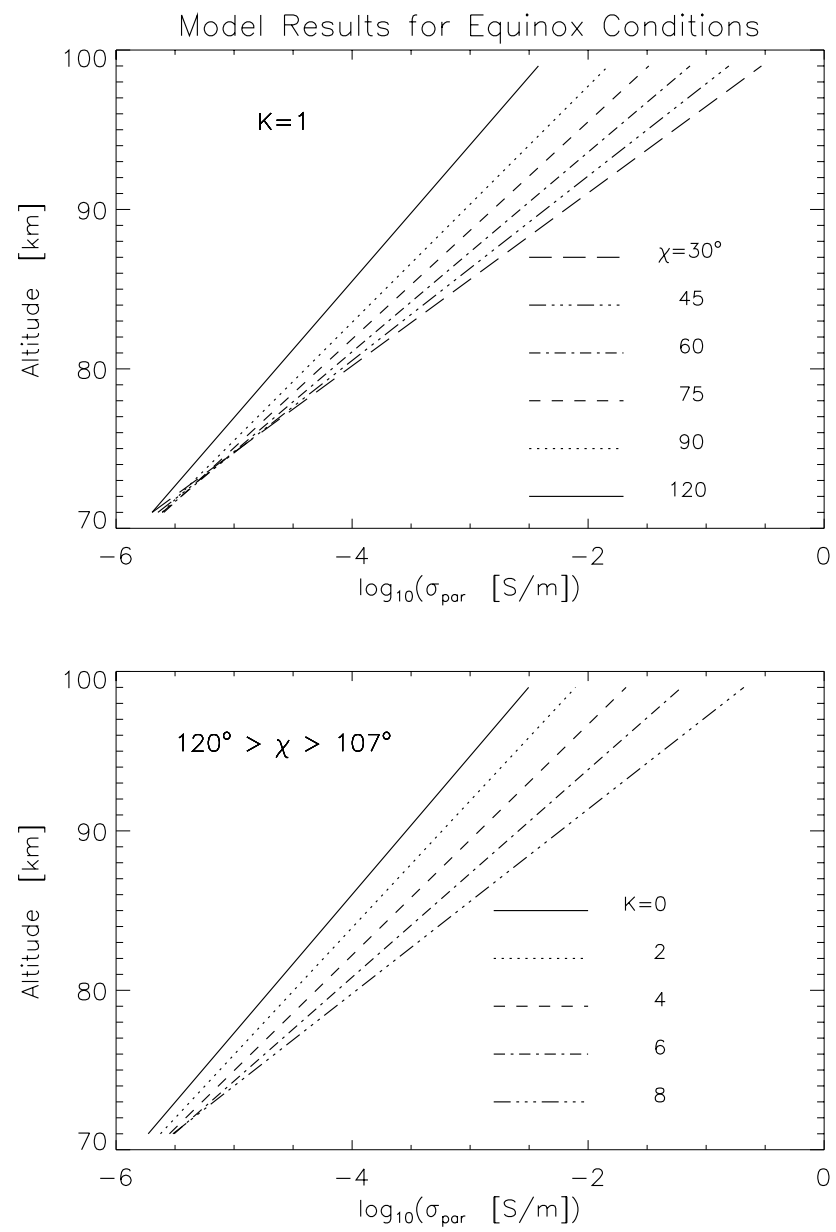

Fig. 7. Examples of conductivity variations with solar zenith angle (upper panel) and with geomagnetic activity (lower panel), both for equinox conditions.

In order to demonstrate this capability, Fig. 7 shows in the upper panel the variation of the conductivity profile for quiet magnetic conditions at equinox, as a function of solar zenith angle. As expected, the variation is very small at altitudes around $70 \mathrm{~km}$, but increases towards greater heights. This is caused by the aforementioned fact that the direct ionization by solar radiation (roughly proportional to $(\cos \chi)^{0.5}$ ) increases with altitude; at low altitudes, the ionization is more controlled by chemistry and transport processes. During equinox the $\chi$-dependence reflects mainly the daily variation of the conductivity profile. In summer/winter (not shown), the curves are shifted slightly towards lower conductivities for higher/lower values of $\cos \chi$ compared to the equinox profiles (cf. Fig. 6).

The lower panel of Fig. 7 shows a similar variation of the conductivity as a function of the geomagnetic disturbance at equinox for a solar zenith angle bin which corresponds to solar heights below the horizon. It represents the conditions in the morning sector of the auroral oval when precipitation is frequent. It is obvious from the comparison of both panels of Fig. 7 that magnetic activity affects the conductivity as strongly as the solar zenith angle. If in addition to a high $K \chi$ also is small, then the profiles are shifted towards even greater conductivities.

Finally, a word about the extrapolation of our model to lower latitudes and the use of $K_{p}$ instead of $K$. Regarding the $\chi$-variation one must be aware of the limitations of our model. It should be remembered that the minimal solar zenith angle in summer at Troms $\varnothing$ is about $46^{\circ}$. An extrapolation to values of $30^{\circ}$ (when used for lower latitudes) may still be realistic, but it becomes doubtful to even smaller values. The model values at low latitudes may thus be regarded as a "qualified guess"; at equatorial latitudes, the usefulness of the model is probably quite uncertain. We see presently no way to test our model in this regime.

The use of $K_{p}$ instead of $K$ is probably justified at latitudes below Scandinavia during quiet and moderately disturbed conditions. During strongly disturbed conditions, heavy particle precipitation (leading to enhanced conductivities) occurs only at auroral latitudes. Our model, if applied to midlatitudes during such conditions, would probably give overestimated conductivities. It should also be kept in mind that our model cannot discriminate between very high activities. Due to the low number of profiles for $K \geq 6$ (Fig. 2), we have lumped together all cases exceeding $K \geq 6$ into one $K$ bin.

It should be mentioned that the WDC for Geomagnetism in Kyoto provides a global conductivity model on the WEB (http://swdedb.kugi.kyoto-u.ac.jp/sigcal/index. $\mathrm{html}$ ), based on the International Reference Ionosphere (http: //nssdc.gsfc.nasa.gov/space/model/models/iri.html) and the neutral atmospheric model CIRA72. Compared to our model it starts at $80 \mathrm{~km}$ altitude (rather than $70 \mathrm{~km}$ as in our case) and uses sunspot number (as proxy for the varying solar activity, rather than $K$ as proxy for the geomagnetic activity in our model) for input. On the other hand, it is laid out as a global model. A comparison for selected cases shows that our conductivities are generally higher than those of the WDC model. This difference can be traced back to considerable deviations of our measured electron density profiles from those of the IRI model. Since our model is based on 11-year measurements at auroral latitudes, it is probably superior for this area. In reality, both models may be used in a complementary fashion. One may also consider using our local model as a calibrator for the global model.

Acknowledgement. We thank the EISCAT Director and staff for running the radar and providing the data. The EISCAT Scientific Association is supported by national scientific organizations of Finland (SA), France (CNRS), Germany (MPG), Japan (NIPR), Norway (NAF), Sweden (NF) and UK (PPARC).

Topical Editor M. Lester thanks M. Friedrich and T. Bösinger for their help in evaluating this paper.

\section{References}

Füllekrug, M.: Schumann resonnances in magnetic field components, J. Atmos. Terr. Phys., 57, 479-484, 1995. 
Füllekrug, M.: Dispersion relation for spherical electromagnetic resonances in the atmosphere, Phys. Lett., A 275, 80-86, 2000.

Lübken, F.-J.: Thermal structure of the Arctic summer mesosphere, J. Geophys. Res., 104, 9135-9149, 1999.

Rishbeth, H. and van Eyken, A. P.: EISCAT: early history and the first ten years of operation, J. Atmos.Terr. Phys., 55, 525-542, 1993.

Schlegel, K. and Füllekrug, M.: Schumann resonance parameter changes during high-energy particle precipitation, J. Geophys. Res., 104, $10111-10118,1999$.

Sentman, D. D.: Schumann resonances, in: Handbook of Atmospheric Electrodynamics, (Ed) Volland, H., Boca Raton, USA, 1, 267-296, 1995.

Schumann, W. O.: Über die strahlungslosen Eigenschwingungen einer leitenden Kugel, die von einer Luftschicht und einer
Ionosphärenhülle umgeben ist, Z. Naturforsch., 7a, 149-152, 1952.

Schunk, R. W. and Nagy, A. F.: Electron temperatures in the Fregion of the ionosphere: Theory and observations, Rev. Geophys. Space Phys., 16, 355-399, 1978.

Schunk, R. W. and Walker, J. C. G.: Theoretical ion densities in the lower ionosphere, Planet. Space Sci., 21, 1875-1896, 1973.

Tran, A. and Polk, C.: The earth-ionosphere cavity, Radio Sci., 11, 803-816, 1976.

Tran, A. and Polk, C.: Schumann resonances and electrical conductivity of the atmosphere and lower ionosphere -1 . Effects of conductivity at various altitudes on resonance frequencies and attenuation, J. Atmos. Terr. Phys., 41, 1241-1248, 1979.

Turunen, T.: GEN system - a new experimental philosophy for EISCAT radars, J. Atmos. Terr. Phys., 48, 777-786, 1986. 